\title{
Convex Hull and Vornoi Diagram based Routing Protocol for Adhoc Network
}

\author{
Buddha Singh \\ Jawaharlal Nehru University \\ New Delhi, \\ India
}

\author{
Silky Makker \\ Manav Rachna International \\ University \\ Faridabad, India
}

\author{
Nisha Tewtia \\ Manav Rachna International \\ University \\ Faridabad, India
}

\begin{abstract}
The MANET faces major challenges in design of routing protocol due to their highly dynamic topology, poses by mobility. The position based routing algorithm that does not depend on topological information is more suitable than other routing protocol for MANET. In previous position based routing protocol does not consider the traffic patterns of higher layer. In fact, the result of these algorithms and test of connectivity in the network layer level and the used to analyze position based algorithm that do not reflect the true behavior of MANET. In our proposed position based routing protocol, we have made modifications in LAR 1 algorithm by changing the request zone region computations. The results of this algorithm are compared with the existing LAR1. The performance of the CHMFR protocols is measured in terms of throughput, success rate and power consumption for varying network size which is efficient as compared to LAR1. We have simulated CHMFR protocol using GloMoSim simulator.
\end{abstract}

\section{Keywords}

Location Aided Routing1(LAR); CHMFR Transport control protocol; Convex Hull, Voronoi Diagram.

\section{INTRODUCTION}

In ad hoc network wireless node that can communicate with each other in the absence of central coordination. In other words in ad hoc networks, autonomous nodes work together to route information through the network. Nodes work as endsystems and routers at the same time. Since nodes work as router, the topology changes dynamically. Therefore, finding and maintaining routes in such network is a non trivial task due to frequent change in topology. We can classify two types of routing protocol on the basis of topological information, Topology based routing and Position based Routing. In topology based routing, nodes must have information of entire topology. If the mobility of nodes is high, there will be frequent path breaks and topological changes will be unpredictable. The second strategy is position based routing in which GPS (Global Positioning System) may require. All position based routing protocols take assumption that the location of host is known.

\subsection{Position-based Routing Protocols}

Routing protocols are used to route a message. Position based routing does not require the maintenance of routes; instead, forwarding decisions are made locally, based only on the node's own position, the positions of its neighbors, and the position of destination. Macker and Corson[10] listed qualitative and quantitative independent metrics for judging the performance of mobile ad hoc networks routing protocols. Desirable qualitative characteristics include: distributed operation, loop-freedom (to avoid energy consumption due to packets spinning around in the network), reactive, and 'sleep period operating (when some nodes become temporarily inactive).

\section{RELATED WORK}

There are several position based routing has been proposed in literature given in [10].

In DIR protocol [14], the source or intermediate node A uses the location information of the destination $\mathrm{D}$ to calculate its direction. Then the message $m$ is forwarded to the neighbor $\mathrm{C}$, such that the $\angle \mathrm{CAD}$ is minimum. The process is repeated until the packet is delivered to the destination.

The message, $\mathrm{m}$ in DREAM protocol [11] is forwarded to the neighbors who are in the direction of destination and also lies between request zones. The request zone determined by the tangents from A to the circle centered at destination. The radius of the expected zone which is the maximal possible movement of $\mathrm{D}$ since the last location update. This can be calculated by multiplying destination's speed to the time elapsed since the last known protocol that uses a limited flooding of location update messages. The experiments show the DIR and DREAM are not loop-free.

In Location Aided routing (LAR) protocol [12], request zone (the area containing the circle and two tangents) is fixed in between the source to destination, and nodes, which are not in the request zone, will not forward a route request to their neighbors. In LAR 2 scheme, the source or an intermediate node A will forward the message to all nodes that are closer to the destination than A. LAR protocol is, essentially, lies in the partial flooding protocol category and flooding is restricted to the request zone. Inside request zone forward the routing packet, the algorithm doesn't optimize the flooding.

In order to reduce flooding rate and provide loop-free behavior the researchers proposed a scheme in which at each intermediate node to destination the expected zone is calculated to find probable successors and to reduce flooding further they applied different methods to select successors node within this expected zone that will forward route request packet towards destination. The message $\mathrm{m}$ is forwarded to exactly those neighbors, which may be the best choices for the possible position of destination (using the distance or progress criterion, respectively).

In VD-GREEDY protocol [1],these neighbors are determined by intersecting the voronoi diagram of neighbors and also lies in the expected zone drawn by the temporary node.

In CH-MFR protocol [1], the neighbor nodes are determined by constructing convex hull of neighbors and selecting the nodes which lies on the boundary of convex hull 
and also lies in the expected zone drawn by the temporary node.

\section{CONVEX HULL AND VORONOI DIAGRAM}

Convex-hull and voronoi diagrams are the concepts of geometry used for determining minimum region that has maximum coverage area required in various applications of mobile network. Some of their applications are routing, cell size, etc. In this section, we give the various definitions of these concepts and the algorithm used to implement them.

\subsection{Definitions}

The standard definitions as cited in $[2,3,13]$ are as follows

\subsubsection{Convexity}

3.1.1.1 Convexity Definition \#1: A set $\mathrm{C}$ is convex if for any $\mathrm{x}$ and $\mathrm{y}$ in $\mathrm{C}$, and for any $\lambda$ between 0 and 1 , the point $\lambda \mathrm{x}+(1-\lambda) \mathrm{y}$ is also in $\mathrm{C}$. that is, if $\mathrm{x}$ and $\mathrm{y}$ are in $\mathrm{C}$, the line segment between $\mathrm{x}$ and $\mathrm{y}$ is completely contained in $\mathrm{C}$. so for example, circles, squares and ovals are convex, but crosses or crescent moons are $\mathrm{n}$ :

3.1.1.2 Convexity Definition \#1: A set $\mathrm{S}$ is convex if given any point $\mathrm{p}, \mathrm{q} € \mathrm{~S}$ any convex combination of $\mathrm{p}$ and $\mathrm{q}$ is in $\mathrm{S}$, or equivalently, the line segment $\overline{\mathrm{pq}} \mathrm{cS}$.

\subsubsection{Hull}

Given a finite set of points $S$ in the plane, a point $p$ in $S$ is hull point if there exists a straight line $\mathrm{L}$ through $\mathrm{p}$ such that all points in $\mathrm{S}$ except $\mathrm{p}$ lie on one side of $\mathrm{L}$ (and except for $\mathrm{p}$, none lies on $\mathrm{L}$ ).

\subsubsection{Convex Hull}

3.1.3.1 Definition \#1: The convex hull $\mathrm{CH}(\mathrm{S})$ of a set $\mathrm{S}$ is the smallest convex set that contains $\mathrm{S}$.

3.1.3.2 Definition \#2: The convex hull $\mathrm{CH}(\mathrm{S})$ of a set $\mathrm{S}$ is the intersection of all convex sets that contain $\mathrm{S}$.

3.1.3.3 Definition \#3: The convex hull $\mathrm{CH}(\mathrm{S})$ of a set $\mathrm{S}$ is the unique convex polygon which contains $\mathrm{S}$ and all of whose vertices are points from $\mathrm{S}$.

3.1.3.4 Definition \#4: For given finite subset of the points $\{\mathrm{p} 1, \mathrm{p} 2 \ldots \mathrm{pk}\}$ a convex combination is any point that can be expressed as a weighted sum:

$\sum_{\mathrm{i}=1}^{\mathrm{n}} \mathrm{CiPi} \quad$ Where $0 \leq \mathrm{ci} \leq 1$ and $\sum_{\mathrm{i}=1}^{\mathrm{k}} \mathrm{Ci}=1$

An equivalent definition of the convex hull is the set of points that can be express as convex combinations of the points in $\mathrm{S}$.

\subsection{Voronoi diagram}

Let $S$ be a set of $n \geq 3$ points $p 1, p 2, p 3 \ldots p n$ in a plane for points $\mathrm{p}=(\mathrm{p} 1, \mathrm{p} 2)$ and $\mathrm{x}=\left(\mathrm{x}_{1}, \mathrm{x}_{2}\right)$ let

$\operatorname{dist}(\mathrm{p}, \mathrm{x})=\sqrt{(\mathrm{P} 1-\mathrm{X} 1)^{2}+(\mathrm{P} 2-\mathrm{X} 2)^{2}}$

denote their Euclidean distance by $\overline{\mathrm{pq}}$ denote the line segment from $\mathrm{p}$ and $\mathrm{q}$. the closure of the set $\mathrm{A}$ will be denoted by $\bar{A}$

\subsubsection{Defination:}

For $\mathrm{p}, \mathrm{q} \mathrm{S}$ let

$\mathrm{B}$ i s t $(\mathrm{p}, \mathrm{q})=\{\mathrm{x} \mid \operatorname{dist}(\mathrm{p}, \mathrm{x})=\operatorname{dist}(\mathrm{q}, \mathrm{x})\}$

$\mathrm{e} \subset \mathrm{B}$ is $\mathrm{t}(\mathrm{p}, \mathrm{q})$

Be bisector of $p$ and $q$. Bist $(p, q)$ is perpendicular line through the center of the line segment pq. It separates the half plane

$\operatorname{dist}(\mathrm{p}, \mathrm{q})=\{\mathrm{x} \mid \operatorname{dist}(\mathrm{p}, \mathrm{x})<\operatorname{dist}(\mathrm{q}, \mathrm{x})\}$

Containing $\mathrm{p}$ from the half plane dist $(\mathrm{p}, \mathrm{q})$ containing $\mathrm{q}$. we can define

$\operatorname{Vr}(p, s)=\bigcap_{q \in g_{1} \neq p} \operatorname{dist}(p, q)$

The voronoi region of $\mathrm{p}$ with respect to $\mathrm{S}$. finally, the Voronoi diagram of $\mathrm{S}$ is defined by

$\mathrm{Vd}(\mathrm{S})=\mathrm{U}_{\mathrm{p}, \mathrm{qE} \in \mathrm{Sq} \neq \mathrm{p}} \overline{\operatorname{Vr}(\mathrm{p}, \mathrm{S})} \cap \overline{\operatorname{Vr}(\mathrm{q}, \mathrm{s})}$

The common boundary of two Voronoi regions belongs to $\mathrm{V}$ (S) and is called a Voronoi edge, if it contains more than one point. If the Voronoi edge e borders the regions of $\mathrm{p}$ and $\mathrm{q}$ then e $<$ Bist $(p, q)$ is satisfied.

\subsection{Algorithm of convex hull and voronoi diagram for static and dynamic network}

The following algorithms [18] have been implemented to compute the radius of a circle drown around the destination in static network and to calculate probable destination on the circle boundary.

Compute - circle - Radius $(\mathrm{P}, \mathrm{D}, \mathrm{N})$ :

1. $\mathrm{n}=$ length $(\mathrm{N})$

2. Create an array Distance $[1 \ldots \mathrm{n}]$

3. For $\mathrm{i}=1$ to $\mathrm{n}$

4. Distance[i] $=\operatorname{dist}(\mathrm{D}, \mathrm{N}[\mathrm{i}])$

5. Sort elements in Distance[1...n] in ascending order

6. $\mathrm{r}=$ Distance $[\mathrm{P} * \mathrm{n} / 100]$

Algorithm1: Pseudo code for computing Radius

Return Convex Hull -Most forward within radius routing algorithm (MFR). Source in this algorithm forward a message. In MFR message is forward to a message to its neighbors that have most forward progress for probable destination. These neighbors for MFR algorithm are determined by using the concept of convex hull for set of nodes of the network. The algorithms for static network [18] are as follows:

$\mathrm{CH}$-Original-Static $(S, D, P, N)$ :

1. $\mathrm{r}=$ Construct-Circle-Static $(\mathrm{P}, \mathrm{D}, \mathrm{R})$

2. Enqueue $(\mathrm{Qmn}, \mathrm{S})$

3. $\mathrm{St}=\mathrm{S}$

4. Stp $=$ St

5. $\mathrm{St}=$ Dequeue $(\mathrm{Qmn})$

6. if(St.R $>=r+$ Dist(st,D))

7. Message delivery is successful

8. else

9. \{

10. if $(\operatorname{Dist}(\mathrm{St}, \mathrm{D})<=\mathrm{r})$

11. \{

12. if ( St and D are connected )

13. Message delivery is successful

14. else 
15. Message delivery is failure

16. $\}$

17. else

18. \{

19. Compute NBts

20. if $(\mathrm{NBts}=\Phi)$

21. Message delivery is failure

22. else

23. \{

24. $\mathrm{CH}=$ Construct-Convex-Hull((NBts)

25. Compute $\mathrm{T} 1$ and $\mathrm{T} 2$ tangents from St on the circle of the radii $r$

26. $\max u=\max y=0$

27. for each ni $€ \mathrm{CH}$ and ni not between $\mathrm{T} 1$ and $\mathrm{T} 2$

28. \{

29. if $(\mathrm{StD} \times \mathrm{Stni} \geq 0)$

30. if ( max $\mathrm{u}<$ projected distance of ni on $\mathrm{T} 1$ )

31. \{

32. $\max \mathrm{u}=$ projected distance of ni on $\mathrm{T} 2$

33. $\mathrm{nu}=\mathrm{ni}$

34. \}

35. else

36. if ( max $\mathrm{v}<$ projected distance of ni on T2)

37. \{

38. $\max \mathrm{v}=$ projected distance of ni on $\mathrm{T} 2$

39. $\mathrm{nv}=\mathrm{ni}$

40. $\}$

41. $\}$

42. for each ni $€ \mathrm{CH}$ and ni is between nu and nv

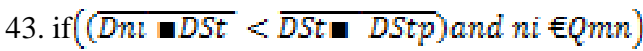

44. Forwarding $[\mathrm{I}]=\mathrm{ni}$

45. $\}$

46. $\}$

This algorithm is based on geographic distance routing (GEDIR). In GEDIR at each hop a source node forwards a message to its neighbors that are closest to the destination. VD GEDIR algorithm modifies the definition of request zone for GEDIR. According to this algorithm a source forwards a message to its neighbors that are closest to a possible position of the destination in expected zone. These neighbors for VDGRDIR algorithm are determined by using the concept of voronoi diagram for set of nodes of network.

\section{FUNCTIONAL IMPLEMENTATION \\ 4.1 Implementation Details}

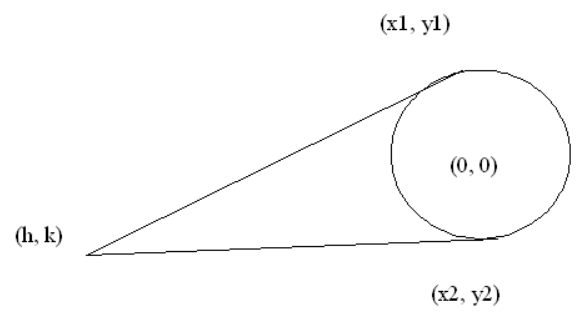

Fig.1 Tangent Drown from external Point

Let the equation of circle is

$x^{2}+y^{2}+2 g x+2 f y+c=0$ with center $(-g,-f)$ and radius $a=\sqrt{g^{2}+f^{2}}-c$ and external points is $(p, q)$.

With this we have

$(x+g)^{2}+(y+f)^{2}=a^{2}$
To calculate the tangential point we will shift the center of the circle form $(-\mathrm{g},-\mathrm{f})$ to $(0,0)$.

Then the equation $(x+g)^{2}+(y+f)^{2}=a^{2}$ will be converted into $\mathrm{x}^{2}+\mathrm{y}^{2}=\mathrm{a}^{2}$.

The co-ordinates of the external point say

$(\mathrm{h}, \mathrm{k})=(\mathrm{p}+\mathrm{g}, \mathrm{q}+\mathrm{f})$

Equation of tangent at circle $x^{2}+y^{2}=a^{2}$ that touches circle at $\left(\mathrm{x}_{1}, \mathrm{y}_{1}\right)$ is $\mathrm{xx}_{1}+\mathrm{yy}_{1}=\mathrm{a}^{2}$.

Since this passes through $(\mathrm{h}, \mathrm{k})$.So $(\mathrm{h}, \mathrm{k})$ will satisfies

$\mathrm{xx}_{1}+\mathrm{yy}_{1}=\mathrm{a}^{2}$

We can get $\mathrm{y} 1$ as follows

$\mathrm{y}_{1}=\frac{a_{2}-\mathrm{nX1}_{1}}{k}$

Since $(x 1, y 1)$ is on the circle $x^{2}+y^{2}=a^{2}$.

We can get a quadratic equation in terms of $x_{1}$

$x 1+\left[\frac{\mathrm{a} 2-\mathrm{hX} 1}{\mathrm{k}}\right]=\mathrm{a} 2$

$$
\left(\mathrm{k}^{2}+\mathrm{h}^{2}\right) \mathrm{x}_{1}^{2}-2 \mathrm{a}^{2} \mathrm{~h}+\mathrm{a}^{2}\left(\mathrm{a}^{2}-\mathrm{k}^{2}\right)=0
$$

Solving this equation we get

$$
=2 \mathrm{ak} \sqrt{\mathrm{k} 2+\mathrm{h} 2-\mathrm{a} 2 \mathrm{a} 2}
$$

Since ( $h, k)$ is out of the circle. So $k^{2}+h^{2}-a^{2} \geq 0$ This implies the value of is real and value of $x_{1}$ will be real and represented as follows

$x 1=\frac{2 a 2 h \pm \mathbf{m}}{2 \cdot(h 2+k 2)}$

Putting this value to the equation of tangent we get the following

$$
\mathrm{y}_{1}=\frac{a_{2}-n x_{1}}{k}
$$

Since for each value of $x_{1}$ we get a value of $y_{1}$. So we have $\left(\mathrm{x}_{1}, \mathrm{y}_{1}\right)$ and $\left(\mathrm{x}_{2}, \mathrm{y}_{2}\right)$ are points then

$(\mathrm{x} 1, \mathrm{y} 1)=\left[\frac{2 \mathrm{a} 2 \mathrm{~h}+\mathrm{m}}{2,(\mathrm{~h} 2+\mathrm{k} 2)}{ }^{x} \frac{\mathrm{a} 2-\mathrm{hX1}}{\mathrm{a} 2}\right]$

And

$(\mathrm{x} 2, \mathrm{y} 2)=\left[\frac{2 \mathrm{a} 2 \mathrm{~h}+\mathrm{m}}{2 \mathrm{a}(\mathrm{h} 2+\mathrm{k} 2)} \frac{\mathrm{a} 2-\mathrm{hX} 2}{\mathrm{a} 2}\right]$

After calculating the value of points we can shift the center to the original point say $(-\mathrm{g},-\mathrm{f})$. Let the final points are $\left(\mathrm{xt}_{1}, \mathrm{yt}_{1}\right)$ and $\left(\mathrm{xt}_{2}, \mathrm{yt}_{2}\right)$. They can be calculated as follows.

$$
\left(\mathrm{xt}_{x} \mathrm{yt} 1\right)=\left[\frac{2 \mathrm{a} 2 \mathrm{~h}+\mathrm{a}}{2 \mathrm{n}(\mathrm{h} 2+\mathrm{k} 2)}-\mathrm{g}, \frac{\mathrm{a} 2-\mathrm{hX12}}{\mathrm{a} 2}-\mathrm{f}\right]
$$

And

$(\mathrm{xt} 2 \mathrm{z} \mathrm{t} 2)=\left[\frac{2 \mathrm{a} 2 \mathrm{~h}+\mathrm{a}}{2 \mathrm{v}(\mathrm{h} 2+\mathrm{k} 2)}-\mathrm{g}, \frac{\mathrm{a} 2-\mathrm{hX} 22}{\mathrm{a} 2}-\mathrm{f}\right]$ 
Now we have an equation of the two tangents say $\mathrm{T}_{1}$ and $\mathrm{T}_{2}$.

$\mathrm{xx}_{\mathrm{t} 2}+\mathrm{yy}_{\mathrm{t} 2}=\mathrm{a}_{2}$

And

$\mathrm{xx}_{\mathrm{t} 1}+\mathrm{yy}_{\mathrm{t} 1}=\mathrm{a}^{2}$

\subsection{Calculation to determine whether a point is inside the request zone}

We know that using computational geometry we can determine whether a point lies left or right to line segment.

\section{Algorithm}

1. Find the tangential points of the circle $t_{1}, t_{2}$ say

$\left(\mathrm{xt}_{1}, \mathrm{yt}_{1}\right)$ and $\left(\mathrm{xt}_{2}, \mathrm{yt}_{2}\right)$.

2. If(! (nt is forward to the intermediate node st))

3. return $(0)$

4. else

5. $\{$ If (nt is in expected zone)

6. return(1);

7. else

8.

9. $\mathrm{D} 1=\operatorname{det}\left(\mathrm{st}, \mathrm{t}_{1}, \mathrm{nt}\right) / /$ calculate determinant value of $\mathrm{nt}$ wrt line

10. $\mathrm{D} 2=\operatorname{det}\left(\mathrm{st}, \mathrm{t}_{2}, \mathrm{nt}\right) / /$ calculate determinant value of $\mathrm{nt}$ wrt line

11. if $((\mathrm{D} 1 * \mathrm{D} 2)<0)$

12. return(1);

13. else

14. return $(0)$;

15. $\}$

16.

17. $\}$

\section{IMPLEMENTATION DETAILS \&} SIMULATION RESULTS

To implement a new protocol into GloMoSim, Therefore it is necessary to describe changes made to the GloMoSim to implement our protocol. Following sections describe the changes made to GloMoSim and analysis of the result obtained.

\subsection{Changes in GloMoSim}

In GloMoSim LAR 1 is the position based routing algorithm available. To implement a new position based algorithm, there are two possible ways. First, implement the new algorithm from the scratch and second one is to make required changes in request zone of existing LAR1.

Second approach is possible since position based routing algorithms in general differ in formation of their respective request zones. In the proposed work we have followed the second approach. In order to implement CHMFR in GloMoSim, we have made the following changes in LAR1:

1) We have modified the LAR1, the purpose of modifying LAR1 is to store information of the nodes that are selected by the algorithm to transmit further, once the packet is received.

means of convex hull while in LAR1 the request zone is a rectangle made of source and destination coordinates.

\subsection{Results \& Analysis}

We have used GloMoSim as simulation tool to test the results cited in [1] in a full network environment. The nodes are spread in terrain of $1000 * 1000$. We have considered network of size 20,30, 50 and 70 nodes and each node has equal transmission range. The nodes are randomly placed within the area. The experiments are repeated 10 times for different seed values for each network size. Mobility is introduced using random way point model.

The performance is measured in the form of matrices for varying network size. The throughput of the protocol is shown in fig 2 .

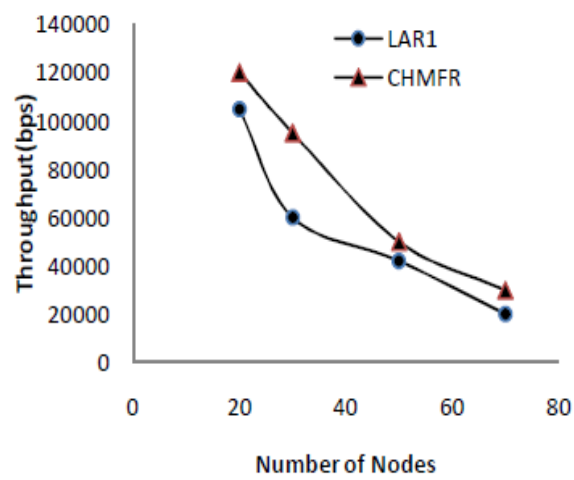

Fig. 2 Throughput of the Network

Fig 2 reveals that as the number of nodes in the network increases, the throughput of the network degrades. The results obtained in CHMFR are more efficient than LAR1. This decline in throughput is caused by collisions and congestion in the network. The fig 2 shows that even after considering the noise, congestion, and collisions CHMFR outperforms LAR1.

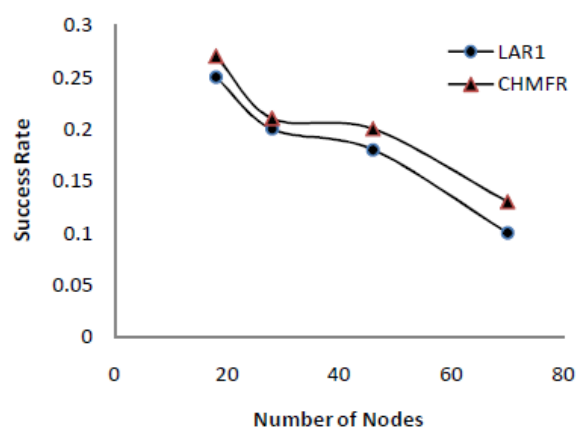

Fig.3 Success Rate of the Network

Fig 3 shows the results of success rate of LAR 1 and CHMFR Success rate is defined as the ratio of total packet received at destination to the total number of packets sent by a source. The authors of [1] have considered that channel is collision free and there is no congestion and path failure among the nodes. Taking noise congestion and interferences between node into consideration, success rate of the CHMFR and LAR1 goes down with the increase of network size. Here again like throughput, CHMFR performs better than LAR1. 
Table 1 shows the power consumption of the network as given below. Power consumption is expressed in terms of the total energy consumed by all the nodes in transmission as well as receiving the packets.

Total Energy consumption $(\mathrm{tp})=\sum_{\mathrm{i}-1}^{\mathrm{n}} \mathrm{pi}$

Where $p_{i}$ is energy consumed by an ith node.

\section{Table1: Power Consumption of LAR1 and CHMFR}

\begin{tabular}{|c|c|c|}
\hline $\begin{array}{c}\text { No of nodes in } \\
\text { network }\end{array}$ & LAR1 & CHMFR \\
\hline 20 & 4503.7706 & 4503.3225 \\
\hline 30 & 6753.9380 & 6753.9851 \\
\hline 50 & 11254.9500 & 11254.7508 \\
\hline 70 & 15764.5210 & 15758.2640 \\
\hline
\end{tabular}

The table shows that even though the power consumption of the network for CHMFR is lesser than LAR1, but the difference is marginal. The success rate of CHMFR is high, however, the number of nodes in the request zone of CHMFR in general is lower than LAR1. Therefore, the number of nodes participating in forwarding the packets is fewer in CHMFR and total energy consumption is less.

\section{CONCLUSION AND FUTURE WORK}

In the current work we have implemented CHMFR; position based routing protocol in performance has been compared with LAR1. Our results obtained shows that the performance of CHMFR outperforms LAR1 as presented by other researchers. However, it is also shown that though the results of these algorithms degrade in a c environment, but the trends of the results remain same. Due to limitations of time we have not implemented voronoi diagram based algorithm as propose in the work. In addition to implement the remaining part of the proposed work, this work can also be extended further the algorithms for other traffic pattern then that CBR. There are certain scopes for improvements in the modified ATP as well. Revision of proposed work may consider highly lossy wireless links causing packet drop rate as high as $10 \%$.

\section{REFERENCES}

[1] Anand Prakash Ruhil', D.K. Lobiya12 and Ivan Stojmenovic3" , Dominating Set based Position Routing in Mobile Ad Hoc Networks.

[2] CMSC 754: Lecture 3 Convex Hulls Tuesday, Feb 6,2006

[3] Franz Aurenhammer, RolfKlien "Voronoi Diagrams".

[4] GloMoSim :manual (ver1.2) http://pcl.cs.ucla..edu/projects/glomosim/GloMoSim Manual.

[5] Ivan Stojmenovic, University of Ottawa and Universidad Nacional Autonoma de Mrxico "Position-Based Routing in Ad Hoc Network" IEEE Communications Magazine, July, 2002.

[6] Ivan Stojmenovic, Anand P.Ruhil, and D.K. Lobiyal, "Voronoi diagram and convex hull based geocasting and routing wireless networks," in Proc. Of Eighth IEEE ISCC, Antalya , Turkey, July 2003, pp. 51-56.

[7] Jorge Nuevo "A Comprehensible GloMoSim Tutorial", INRSUniversite du Quebec nuevo@ins-telecom. uquebec.ca, March 4,2004.

[8] Lili Zhang, "Position-Based Routing", in Algorithms for adhoc networking - seminar talk of 29th september 2003.

[9] Martin Mauve, Jorg Widmer, and Hmnes Hartenstein, “A servey on position=Based Routing in Mobile Ad Hoc Networks" IEEE Network, pp, 30-39,Nov/Dec 2001. 